Revista de Psicología Vol. 36 (2), 2018 (ISSN 0254-9247)

\title{
Adaptación de la Escala de Valoración de la Conducta Violenta y Riesgo de Reincidencia (EVCV-RR) en una muestra penitenciaria peruana ${ }^{1}$
}

\author{
Jari Yasser Conde Mendoza² \\ Universidad Nacional de San Agustín de Arequipa, Perú
}

\begin{abstract}
Los sistemas judiciales exigen a los psicólogos evaluar a sujetos que cumplen condenas en establecimientos penitenciarios con el fin de valorar la posibilidad que estos puedan reinsertarse anticipadamente a la sociedad. Para ello, los psicólogos penitenciarios han desarrollado técnicas y procedimientos de evaluación del riesgo de la conducta violenta basadas en la identificación de factores de riesgo. El objetivo de este estudio fue adaptar el primer instrumento de evaluación del riesgo de violencia al contexto penitenciario peruano. Se estableció la validez de contenido por juicio de expertos, con una muestra de 303 internos. La validez de constructo fue establecida a través del análisis factorial exploratorio y confirmatorio. Por último, se estableció la precisión diagnóstica, por medio de la curva ROC. Los resultados indican que la adaptación de la EVCV-RR es un instrumento adecuado para valorar el riesgo de criminógeno.

Palabras clave: psicología forense, análisis factorial, riesgo de violencia, riesgo criminógeno.
\end{abstract}

Adaptation of the Evaluative Scale of Violent Behavior and Risk of Recidivism (SVVBRR) in a Peruvian penitentiary sample

The judicial systems require psychologists to evaluate subjects who serve sentences in penitentiary establishments in order to assess the possibility of reinsertion in society. Consequently, prison psychologists have developed techniques and procedures for assessing the risk of violent behavior based on the identification of risk factors. The objective of this study was to adapt the first instrument for assessing the risk of violence in the Peruvian prison context. Content validity was evidenced by expert judgment and with a sample of 303 inmates, construct validity was identified through exploratory and confirmatory factor analysis. Finally, the diagnostic accuracy was established by means of the ROC curve.

1 Esta investigación fue financiada por la Universidad Nacional de San Agustín de Arequipa, según contrato de Subvención Nº71-2016-UNSA.

2 Maestría en Psicología Jurídica y Forense de la Universidad Nacional de San Agustín de Arequipa. Dirección postal: Av. Venezuela s/n - Pab. Psicología R.I.P- CC 2do. piso - Arequipa, Perú. Contacto: jariconde.m@gmail.com 
The results indicate that the adaptation of the EVCV-RR is an adequate instrument to assess the criminogenic risk.

Keywords: Forensic Psychology, factorial analysis, risk of violence, criminogenic risk.

\section{Adaptaçáo da Escala de Avaliaçáo de Comportamento Violento e Risco de Recorrência (EACV-RR) em uma amostra de penitenciária peruana}

Os sistemas judiciais exigem que os psicólogos avaliem assuntos que atendam sentenças em estabelecimentos penitenciários, a fim de avaliar a possibilidade de serem reinseridos antecipadamente para a sociedade. Para isso, os psicólogos da prisáo desenvolveram técnicas e procedimentos para avaliar o risco de comportamento violento com base na identificação de fatores de risco. O objetivo deste estudo foi adaptar o primeiro instrumento para avaliar o risco de violência ao contexto prisional peruano. A validade do conteúdo foi evidenciada por julgamento perito e com uma amostra de 303 presos, a validade de construçáo foi identificada através de análise fatorial exploratória e confirmativa. Finalmente, a precisão diagnóstica foi estabelecida por meio da curva ROC. Os resultados indicam que a adaptação do EVCV-RR é um instrumento adequado para avaliar o risco criminoso.

Palavras-chave: Psicologia Forense, análise fatorial, risco de violência, risco criminoso.

\section{Adaptation de l'Échelle d'Évaluation du Comportement Violent et du Risque de Récur- rence (EECV-RR) dans un échantillon pénitentiaire péruvien}

Les systèmes judiciaires exigent des psychologues qu'ils évaluent les sujets qui purgent des peines dans les établissements pénitentiaires afin d'évaluer la possibilité qu'ils soient réinsérés à l'avance dans la société. Pour cela, les psychologues de prison ont développé des techniques et des procédures pour évaluer le risque de comportement violent basé sur l'identification des facteurs de risque. L'objectif de cette étude était d'adapter le premier instrument d'évaluation du risque de violence au contexte pénitentiaire péruvien. La validité du contenu a été mise en évidence par un jugement d'expert et, avec un échantillon de 303 détenus, la validité du concept a été identifiée par une analyse factorielle exploratoire et confirmatoire. Enfin, la précision du diagnostic a été établie au moyen de la courbe ROC. Les résultats indiquent que l'adaptation de l'EECV-RR est un instrument adéquat pour évaluer le risque criminogène.

Mots-clés: Psychologie médico-légale/judiciaire/criminelle, analyse factorielle, risque de violence, risque criminogène. 
Los operadores de justicia alrededor del mundo exigen a los profesionales de la psicología predecir la conducta violenta de aquellos sujetos que violaron las leyes y que podrían volver a tener estas conductas que afectan directamente a la sociedad. Para los psicólogos forenses, encontrar una forma eficaz y eficiente de dar una conclusión sobre esta problemática ha sido siempre una tarea ardua y difícil, ya que esta labor puede proteger o poner en riesgo a todos los miembros de una sociedad (Jiménez, Sánchez, Merino \& Ampudia, 2014b; Martínez, 2014).

Diversos investigadores han desarrollado desde hace décadas múltiples métodos y técnicas para identificar a un sujeto con diverso grado de riesgo de conducta violenta (Douglas, Hart, Webster \& Belfrage, 2013; Hare, 2003; Hanson, 2004; Hanson \& Thornton 2000; Jiménez, Sánchez, Merino \& Ampudia, 2014b; Lewis, Olver \& Wong, 2016; Lucioni \& Pueyo, 2015; Monahan et al., 2005; Monahan, 2008; Quinsey, Harris, Rice \& Cormier, 1998, 2006; Schuringa, Heininga, Spreen \& Bogaerts, 2016; Webster, Douglas, Eaves \& Hart, 1997). Estos investigadores, a través de grandes recolecciones de datos han identificado múltiples factores de riesgo estáticos y dinámicos (Conroy $\&$ Murrie, 2007) que han ido formando una base sólida para crear diversos instrumentos para la valoración del riesgo.

La evaluación de la conducta violenta en sujetos dentro de contextos forenses y penitenciarios ha ido evolucionando desde predecir la peligrosidad, hasta valorar el riesgo de violencia de un sujeto (Conroy \& Murrie, 2007; Jiménez et al., 2014a). Para entender el proceso de evaluación del riesgo de una conducta violenta, hay que tener en cuenta el término peligrosidad y dos conceptos fundamentales: el riesgo y la evaluación de riesgo.

El termino peligrosidad, en el ámbito forense, se centra en las características de personalidad del sujeto, pero como un concepto dicotómico "peligroso / no peligroso". La peligrosidad evaluada de esta 
forma resulta deficiente en el campo forense (Heilbrun, 2009), ya que este término da pie a que al perito en psicología forense se le pregunte en audiencia; "Dígame usted, ¿este sujeto es peligroso?" "respóndame, ¿sí o no?”. Se tiene muchos testimonios de profesionales que laboran en centros penitenciarios que indican que no pueden dar y sobre todo que no deberían dar respuesta a esta pregunta cerrada, es por eso que como respuesta ellos indican en audiencia: "no puedo contestar a esta pregunta; el comportamiento humano es dinámico y no dicotómico", "yo no puedo decir si es sí o no... yo me baso en las probabilidades que me hace concluir la evaluación hecha al sujeto". Incluso se tiene conocimiento que muchos profesionales han enfrentado a jueces y fiscales por no dejarlos leer o explicar la evaluación que realizaron y simplemente reducirlos a una pregunta sin lógica alguna, limitando la importante contribución del profesional al no dejar que el psicólogo pueda dar su opinión sobre el riesgo de la conducta violenta del sujeto y como es que el tratamiento penitenciario influyó en el sujeto durante su internamiento (Kroner, 2005).

El pronosticar algo o predecir algún suceso (e. g., sobrevivir a una intervención quirúrgica al corazón) se debe expresar en probabilidades. Al hablar de la peligrosidad, no podemos decir que alguien sea simplemente "peligroso" o "no peligroso"; al usar distintos niveles de estimaciones del riesgo, es decir; bajo, medio y alto, se disminuyen los errores de sobreestimación del riesgo (Conroy \& Murrie, 2007; Jiménez et al., 2014b; Muñoz \& López, 2016), ya que se mide un constructo de forma continua, el cual se puede entender mejor probabilísticamente (Helmus \& Babchishin, 2017).

Debemos tener presente también que la información que se toma para evaluar la llamada peligrosidad es muy similar a cuando se valora el riesgo de una conducta violenta; la diferencia claramente está en cómo se organiza y se determina la información crucial para hacer una correcta evaluación del riesgo de violencia (Pueyo \& Redondo, 2007). Parece que el utilizar el modelo de evaluación del riesgo de violencia ha significado el acierto más representativo que se ha obtenido al evaluar la probabilidad de conducta violenta en los últimos años (Kroner, 2005). 
Por otra parte, uno de los conceptos fundamentales que debemos entender es el riesgo. El riesgo es la probabilidad de que suceda algo y, para el caso específico de una conducta violenta, que alguien resulte afectado, provoque a otra persona o a uno mismo un daño (Jiménez et al., 2014b). El termino riesgo representa un concepto complejo y situacional que va a depender de su naturaleza, probabilidad, gravedad, además otras circunstancias especiales (Lucioni \& Pueyo, 2015).

Otro concepto fundamental e incluso más importante, es la "evaluación del riesgo", este proceso metódico a través del cual se valora la probabilidad de que un sujeto tenga un comportamiento violento, en este caso delictivo (Singh, 2012), implica la recuperación de información de un sujeto y de su entorno global (Department of Family and Community Services, 2015). Este procedimiento se basa en la identificación de condiciones especiales y especificas relevantes a través de las cuales se pueden conocer los factores de riesgos de un sujeto (Brown $\&$ Singh, 2014; Arbach-Lucioni et al., 2015).

La valoración del riesgo, dentro del ámbito penitenciario, sirve especialmente a la hora de clasificar a un sujeto por niveles de seguridad a través del riesgo de violencia que pueda presentar, y sobre todo ofrecer un argumento científico para ayudar a los jueces en la toma de decisiones sobre el otorgamiento o negación de un beneficio penitenciario como es la semi-libertad o libertad condicional (Andrade, O'neill \& Diener, 2009; Fazel, Singh, Doll \& Grann, 2012; Skeem \& Monahan, 2011).

Ahora bien, debemos aclarar que el término valoración del riesgo de violencia es tomado para la práctica penitenciaria peruana como valoración del riesgo criminógeno (Decreto legislativo 1328, artículo 30), siendo ambos términos equivalentes, ya que utilizan la misma metodología al momento de hacer la valoración referida a la conducta de riesgo de un interno.

Existen diversos criterios metodológicos para evaluar el riesgo de una conducta violenta, entre estos métodos los más importantes y respaldados por la práctica son: el método actuarial, el método clínico estructurado y la evaluación de riesgo estructurado (Monahan, 2008). 
El método actuarial utiliza la evaluación objetiva, apoyado en un conjunto de algoritmos matemáticos (Hart, Michie, Cooke, 2007; Heilbrun, 2009); evalúa la interacción de factores y brindan una conclusión del riesgo del sujeto sin necesidad que intervenga un psicólogo o evaluador con amplia experiencia (Fuster, 2014). El método actuarial, se centra en los factores de riesgo históricos o estáticos (Andrade, O'neill \& Diener, 2009). Por "factores de riesgo estáticos" se entiende que son diversas situaciones o condiciones sociales y culturales de muy difícil modificación, situaciones o condiciones biológicas, psicológicas de evolución del individuo (e. g., constante maltrato desde la infancia) y psicopatológicas irreversibles o de diagnóstico negativo (Andrews, Bonta \& Wormith, 2006; Singh, 2012). El principal problema de este método es que no toma en cuenta la dinámica de variación que puede tener un sujeto (e. g., recibir un tratamiento psicológico, cambio de contexto, aprendizaje educativo, etc.) luego de ser evaluado bajo este método, aunque instrumentos actuariales actualizados si incorporan factores dinámicos en la evaluación (Andrade, O’neill \& Diener, 2009).

Por otra parte, el juicio clínico estructurado se desarrolló para dar flexibilidad al método actuarial (Neal \& Grisso, 2014). Este método ayuda a los expertos penitenciarios a identificar por medio de una valoración para cada factor de riesgo examinado, asignando una valoración final categórica (bajo riesgo, moderado riesgo y alto riesgo) (Brown \& Singh, 2014) a través de la presencia y la ausencia de factores de riesgo y teniendo en consideración los factores de riesgo dinámicos en su mayoría (además de los factores de riesgo estáticos). Se entiende por "factores de riesgo dinámicos" a todas aquellas condiciones biológicas, psicológicas, sociales y culturales de posible cambio (e. g., distorsiones cognitivas, creencias, situación de desempleo, falta de recursos educacionales, psicopatología de diagnóstico favorable y otros) (Andrews et al., 2006; Singh, 2012). El juicio clínico estructurado a primera impresión parece ser más realista al evaluar el riesgo de un sujeto (Muñoz \& López, 2016), pero a la vez demuestra ser menos objetivo, cargado de sesgos y de la subjetividad del evaluador (Brown \& Singh, 2014); 
por eso es que el profesional debe de tener un amplio conocimiento en conductas violentas y de factores de riesgo delictivos.

El juicio clínico estructurado (Monahan, 2008; Wong, Olver \& Stockdale, 2009) sigue un procedimiento explicito para la identificación y evaluación de factores de riesgo, este procedimiento puede introducir puntuaciones, o no, para poder determinar la valoración final de la evaluación de riesgo (Pueyo \& Redondo, 2007).

Las diferencias entre ambos modelos de evaluación indican que en la práctica no son distintos significativamente a la hora de valorar el riesgo de violencia, es decir, muestran un corto margen de diferencias en la capacidad predictiva entre métodos (Fazel, Singh, Doll \& Grann, 2012; Singh, Fazel, Gueorguieva \& Buchaman, 2014).

La combinación entre el método actuarial y el método clínico estructurado se denomina "Evaluación de riesgo estructurado" (Monahan, 2008), y resulta una de las mejores prácticas para la evaluación del riesgo de una conducta violenta (Heilbrun, 2009; Pueyo \& Arbach-Lucioni, 2015). Ambos modelos se implementan metódicamente en instrumentos estandarizados, protocolos estandarizados, en guías estructuradas o en todo caso en la práctica del forense (Quinsey, Harris, Rice \& Cormier, 2006). Esta idea se refuerza con el planteamiento de que el profesional forense debe seguir procedimientos rigurosamente científicos; es decir, realizar una práctica basada en la evidencia (Fernández-Ballesteros, Oliva, Vizcarro \& Zamarrón, 2011).

En suma, todas las acciones que se realicen para regular una situación de riesgo de una conducta violenta. En otras palabras, la obtención de información, el uso de técnicas, métodos aunados y estrategias, es a lo que se le denomina "gestión de riesgo" (Lucioni \& Pueyo, 2015).

Existen estudios que han identificado los principales factores de riesgo asociados a una conducta violenta: la edad del sujeto, que muestra una relación inversa con la conducta violenta, a menor edad del sujeto, mayor riesgo de una conducta violenta, y a edades mayores el riesgo disminuiría (Harris \& Rice, 2007; Instituto Nacional Penitenciario, 2016; Louw, Strydom \& Esterhuyse, 2005). El grado de instrucción también tiene una relación inversa parecida a la edad con el 
riesgo de una conducta violenta (Bonta, Law \& Hanson, 1998; INPE, 2016; Louw, Strydom \& Esterhuyse, 2005).

Por otra parte, están los comportamientos y circunstancias desajustadas durante la infancia, que son predecesores de una conducta violenta por circunstancias como disfuncionalidad familiar y social, falta de atención en el medio escolar, repitencia de años escolares, suspensión o expulsión del colegio, impulsividad, problemas con sus pares, padres excesivamente disciplinados o que tienen a sus hijos en abandono, ausencia de padres, conductas antisociales, consumo de alcohol o drogas, aparición de enfermedad mental en la infancia y otros (Louw et al., 2005; Petrosino, Derzon \& Lavenberg, 2009; Schick \& Cierpka, 2016). Las características de personalidad también son un factor de riesgo importante (Willmot, 2004) que podrían conllevar a diversas situaciones de conflicto debido al poco control de emociones, a un estilo cognitivo rígido, egoista, irresponsable, etc. (Pueyo \& Redondo, 2004).

Un historial violento, como factor de riesgo, es uno de los mejores predictores de una conducta violenta futura (Grann, Belfrage \& Tengström, 2000; Louw et al., 2005; Monahan et al., 2001). Es decir, una conducta violenta de inicio en la infancia o adolescencia que ha sido mantenida hasta la adultez es considerada como una carrera delictiva (Monahan, 2003), aunque no todas las conductas violentas de un sujeto están identificadas en un historial. Por ello, la experiencia del forense debe de ser suficiente para identificar conductas violentas paralelas a las que están en historiales, entrevistas al interno, entrevistas a personas cercanas y fuentes terciarias (Andrade, O'neill \& Diener, 2009; Heilbrun, 2009).

La psicopatía como factor de riesgo, al igual que un historial violento, es un gran predictor de una conducta violenta futura, siendo tal vez estos dos factores de riesgo los que más pueden contribuir en la valoración de riesgo de violencia (Tengströn, Hodings, Grann, Langström \& Kullgren, 2004). Diversas investigaciones han clasificado a la psicopatía como un factor estático, debido a la poca respuesta positiva al tratamiento que muestran los sujetos identificados como psicópatas (Conroy \& Murrie, 2007; Otto, 2000). 
Otro factor estrechamente relacionado con el riesgo de una conducta violenta es el consumo de drogas (Conroy \& Murrie, 2007; Jiménez et al., 2014b; Louw et al., 2005; Willmot, 2004). El consumo excesivo de alcohol y otras sustancias producen deterioros crónicos y desarrollan síntomas psiquiátricos, además de estar relacionado significativamente con los suicidios, el maltrato infantil y los abusos sexuales. Igualmente, el consumir alcohol y otras sustancias sin tener una adicción puede llevar a tener conductas agresivas y violentas (Tardiff, 2008).

Por último, uno de los factores de riesgo, relacionados con el riesgo de violencia, es la violación de medidas de supervisión (Conroy \& Murrie, 2007). En general, un historial de dificultades para continuar con el tratamiento penitenciario, actitudes negativas hacia el tratamiento, incumplimiento de reglas de conducta dentro del establecimiento penitenciario e incumplimiento con reglas de conducta de un sujeto, habiéndosele concedido la semi-libertad o libertad condicional, son indicadores altamente relacionados con una conducta violenta reincidente (Lucioni \& Pueyo, 2015).

Los factores de riesgo dinámicos y estáticos son vertidos en protocolos, escalas, cuestionarios y guías de valoración del riesgo de violencia de amplio uso alrededor del mundo; entre ellos están el Violence Risk Appraisal Guide-Revised (VRAG-R) de Rice, Harris \& Lang (2013); la Psychopathy Checklist-Revised (PCL-R, 2da. Ed.), de Hare (2003); el Rapid Risk Assessment for Sexual Offense Recidivism (RRASOR), de Hanson (2004) y el Historial, Clinical, Risk Management-20, (HCR-20 v.3) de Douglas, Hart, Webster \& Belfrage (2013).

Un instrumento innovador que contiene ítems específicos de la PCL-R, del HCR-20 y del VRAG, es la Escala de Valoración de la Conducta Violenta y Riesgo de Reincidencia, (EVCV-RR) de Jiménez et al., (2014b), validada inicialmente con una muestra espańola y actualmente en desarrollo con una adaptación a la población penitenciaria mexicana. Esta escala cuenta con 27 ítems de evaluación específica para personas internadas en centros penitenciarios y evidencia información sobre tres variables específicas: violencia, psicopatía y reincidencia. A la lista, se suman docenas de instrumentos desarrollados, adaptados e investigados 
en el mundo; todos ellos enfocados en deslumbrar nueva evidencia que ayude a valorar el riesgo de la conducta violenta de un sujeto.

Por otra parte, desde los años 90 en Latinoamérica, los objetivos referidos a la evaluación del riesgo de la conducta violenta recaía sobre el termino peligrosidad (si/no), los factores de riesgo dinámicos, la necesidad del aumento de investigaciones que identificaran específicamente diversos factores de riesgo, la especificidad de los pronósticos, la utilización de diversas fuentes de información y la prevención en la sociedad (Folino, Sarmiento \& Montero, 2000; Folino, 2000). La mayoría de investigaciones de este tipo se realizaron en Argentina, Brasil, Chile y Colombia (De Borba, Folino \& Taborda, 2009; Tapias, 2011; Telles, Day, Folino \& Taborda, 2009).

Una de estas investigaciones fue la de Singh, Condemarín \& Folino (2013) realizada a 46 profesionales de la salud mental forense en Chile y Argentina evidenciando que un 93\% de los psicólogos de la muestra argumentaron la gran importancia y mayor utilidad del HCR-20 y la PCL-R teniendo este último instrumento diversas adaptaciones en varios países de América (León, Asún \& Folino, 2010; Folino \& Hare, 2005; Medina, Valdés, Galán, Vergara \& Couso, 2013; Moltó, Poy \& Torrubia, 2000; Ostrosky, Ruiz, Arias \& Vásquez, 2008; Ruiz, 2006). Folino (2015) realizó una de las más recientes investigaciones en Latinoamérica referida a la validez, predictividad y la reincidencia de una conducta violenta utilizando diversos instrumentos como el PCL-R, el VRAG, el HCR-20 y el juicio clínico estructurado.

En el Perú solo se encontró una escala adaptada a la población penitenciaria (mujeres) realizada por Loyola (2011). En su trabajo investigo la validez y la confiabilidad de la Escala de Impulsividad Barratt (BIS11) en dos establecimientos penitenciarios en la ciudad de Lima con muestra conformada por 216 mujeres. Teniendo en cuenta que en Perú no existe una medida estructurada para la realidad penitenciaria y específicamente para la valoración del riesgo criminógeno, es propósito de esta investigación desarrollar la adaptación de la Escala de Valoración de la Conducta Violenta y Riesgo de Reincidencia (EVCV-RR) para poder fortalecer las pericias psicológicas en el contexto penitenciario y forense. 


\section{Método}

\section{Participantes}

La adaptación de la EVCV-RR se realizó en un establecimiento penitenciario de varones en Arequipa; la muestra fue seleccionada a través del método probabilístico estratificado (Hernández, Fernández \& Baptista, 2014) y estuvo conformada por 384 internos (diversos delitos), que tenían la situación jurídica de sentenciados.

Para la prueba piloto (PP) participaron 81 internos los cuales tenían entre 21 y 64 años de edad $(M=37.31, D E=10.71)$, siendo identificados por la junta técnica de clasificación del E.P. 36 de ellos como violentos y 45 como no violentos; una proporción de 3.5/1 (ítems por sujeto) para los 23 ítems inicialmente computables, lo que sería el mínimo necesario para la PP (Lloret-Segura, Ferreres-Traver, Hernández-Baeza \& Tomás-Marco, 2014).

Para el presente estudio, la muestra para la aplicación de la prueba propiamente dicha (PPD), estuvo conformada por 303 internos los cuales tenían entre 18 y 71 años $(M=36.02, D E=10.56)$, siendo identificados 154 internos (50.83\%) como violentos y 149 internos (49.17\%) como no violentos; con una proporción de 13/1 para los 23 ítems inicialmente computables, estando dentro de la cantidad de muestra adecuada para el AF (Beavers et al., 2013; Byrne, 2010; Costello \& Osborne, 2003; Kline, 2011; Mvududu \& Sink, 2014). Los internos de la PPD fueron distintos a los de la PP y a todos se les leyó un consentimiento informado y posteriormente se firmó, por y para cada parte, (entrevistador y entrevistado), un acta de consentimiento informado que resumía el consentimiento informado.

\section{Medición}

El instrumento a adaptar fue la Escala de Valoración de la conducta violenta y riesgo de reincidencia (EVCV-RR) (Jiménez, Sánchez, Merino \& Ampudia, 2014a). La EVCV-RR es una entrevista guiada estructurada que se combina con la información del expediente del 
establecimiento penitenciario. Utilizando metodología actuarial y clínica, dicha escala se compone de un total de 27 ítems (17 computables) con una puntuación según sigue: 4 ítems refieren distintas variables socio-biográficas (1 al 4.), 10 ítems hacen referencia a la problemática específicamente carcelaria (ítems 5 al 13 y el 27) y los 13 restantes son considerados como variables específicas de la psicopatía. Los ítems se califican en una escala ordinal ( 0 = nada; 1 = algo; 2 = bastante; 3 = mucho). La puntuación final permite valorar el riesgo de conducta violenta, reincidencia y psicopatía; "Muy baja”, "Baja”, "Media”, "Alta" y "Muy alta" (Jiménez et al., 2014a).

La EVCV-RR, está basada en instrumentos de valoración del riesgo de amplia investigación pudiendo ayudar a los psicólogos a predecir y evaluar la conducta violenta y el riesgo de reincidencia. El desarrollo de la escala original fue conformado por una muestra de 49 internos en un centro penitenciario, siendo 21 de ellos considerados como violentos y 28 como no-violentos. La consistencia interna fue de $(\alpha=.835)$ y la precisión diagnóstica a través de la curva ROC, resultó ser AUC = .904 y la diferencia de las puntuaciones medias obtenidas por los dos grupos, peligrosos/no-peligrosos, obtuvieron un buen índice de Cohen $(d=1.70)$ (Jiménez et al., 2014a). El permiso de adaptación se solicitó al profesor Fernando Jiménez Gómez, que aprobó la adaptación de la escala.

\section{Procedimiento}

El proceso de adaptación de la EVCV-RR se dividió en cinco etapas: la validez de contenido, la prueba piloto (PP), el análisis factorial exploratorio de la prueba propiamente dicha (PPD), el análisis factorial confirmatorio de la PPD y precisión diagnostica. La primera etapa, validez de contenido, se realizó por juicio de expertos que laboraban en un establecimiento penitenciario de varones en Arequipa el momento de realizar el juicio de expertos, y tenían amplia experiencia de trabajo dentro de diversos establecimientos penitenciarios del Perú. Para el análisis de datos se utilizó el coeficiente V de Aiken (Aiken, 2003; Merino \& Livia, 2009; Rodríguez, 2014). 
La segunda etapa de la prueba piloto (PP) se realizó para evitar inconvenientes que sean difíciles de resolver en la PPD, dilucidar dudas de cómo abordar la entrevista e identificar la impresión del evaluador, identificar errores no percibidos al redactar y revisar la guía, identificar el tiempo de demora promedio al utilizar la guía de entrevista y obtener los primeros resultados de análisis de los ítems que direccionen y den sentido a la escala.

Para la tercera etapa se realizó el análisis factorial exploratorio de la prueba propiamente dicha (PPD). Se verificó la existencia de datos ausentes y posteriormente se evidenció los casos de datos atípicos para puntuaciones estandarizadas (Tabachnick \& Fidell, 2001). El método de box plots identificó datos atípicos similares a los de las puntuaciones estandarizadas; se decidió retener los casos atípicos para experimentar y confirmar si se produce una afectación importante en la estructura factorial de la adaptación de la EVCC-RR para la PPD.

Se procedió a evidenciar la distribución de las variables computables a través de los estadísticos de asimetría y curtosis (Bandalos \& Finney, 2010; Gaskin, 2016; George \& Mallery, 2003; Lloret-Segura, Ferreres-Traver, Hernández-Baeza \& Tomás-Marco, 2014), y se utilizaron los ítems puntuados para elaborar los análisis. Con el análisis factorial exploratorio se utilizó el método de factorización de ejes principales debido a la variación leve de normalidad de los datos de la muestra y la rotación oblicua "promax" estimándose posteriormente la consistencia interna por el método Alfa de Cronbach (Batista, Coenders \& Alonso, 2004; Romero \& Ordoñez, 2015).

En la cuarta etapa se realizó el análisis factorial confirmatorio para la PPD y precisión diagnostica. Se procedió a observar el índice de curtosis multivariante y su relación critica (Mardia, 1985). Se especificó e identificó el modelo fijándose tres pesos de regresión para cada grupo de variables con la constante 1, que para Arbuckle (2014), es suficiente para identificar el modelo. El método de estimación utilizado fue el de máxima verosimilitud y se consideraron diversos índices de ajuste, como el CMIN/df, RMR, GFI, NFI, TLI, CFI y el RMSEA, que brindan la información necesaria sobre la determinación de modelo 
especificado sobre los datos de la muestra (Hu \& Bentler, 1998). El modelo propuesto inicialmente se rechazó y se reespecificó basado en los pesos de regresión estandarizados, las correlaciones múltiples al cuadrado, los índices de modificación y los índices de ajuste del modelo.

En la última etapa, la evaluación de la precisión diagnostica de la adaptación de la EVCV-RR se midió a través de la curva ROC (Receiver Operating Characteristic curve - ROC curve) (Hanson, 2009; Howard, 2017; Singh, Desmarais \& Van Dorn, 2013; Steyerberg et al., 2010), utilizando como referencia la identificación como, violento/no-violento, que fue concluida por la junta técnica de clasificación del establecimiento penitenciario.

\section{Análisis de datos}

Para adaptación de la EVCV-RR se realizó un análisis factorial exploratorio tanto como para la prueba piloto y para la prueba propiamente dicha, utilizándose el software SPSS v.24 el cual permitió evidenciar la estructura factorial exploratoria de la escala para que posteriormente sea analizada a través del análisis factorial confirmatorio para el cual se usó el software de interfaz gráfica AMOS v.24. Finalmente, la precisión diagnostica de la escala se midió a través del software MEDCALC v.17.2, evidenciando adecuados índices para el modelo final de la adaptación peruana de la EVCV-RR.

\section{Resultados}

\section{Validez de contenido}

La primera parte del juicio de expertos la realizaron 14 expertos penitenciarios (siete psicólogos, dos abogados, dos trabajadoras sociales); se construyeron dos formatos especiales para la evaluación de los 27 ítems de la escala. Los criterios para la evaluación fueron: suficiencia, claridad, redacción, coherencia y relevancia. Además, ofrecieron su opinión y sugerencias para cada ítem y su forma de entrevista. Se estimó el acuerdo del juicio de expertos, con la $\mathrm{V}$ de Aiken y se evidenció que 
los ítems 1, 2, 4, 8, 9, 11, 22 y 24, no superaban el .80 como límite (Rodríguez, 2014); por lo tanto estos ítems fueron modificados necesariamente en su contenido. La segunda parte del juicio de expertos la realizaron 3 expertos penitenciarios (una psicóloga, un abogado y una trabajadora social) diferentes a los de la primera parte. Se utilizaron los mismos criterios y procedimientos de la primera parte y se evidenciaron índices de V de Aiken superiores al .97 en todos los ítems.

\section{Prueba piloto (PP)}

La prueba piloto sirvió para reducir inconvenientes que podían traer dificultades en la PPD, ayudó a desarrollar un mejor abordaje de la entrevista y evidenció la impresión del evaluador. Asimismo, aseguró que el contenido de los ítems fuera el adecuado e identificó errores que solo se podían evidenciar en la práctica de la entrevista y con diversos entrevistados; se determinó un promedio de 30 minutos de demora para la evaluación total de los ítems. Además, se obtuvo la estructura factorial a través del método de ejes principales con rotación "promax". La estructura factorial dio pie a pensar que los ítems se ordenaban de manera adecuada para cada variable, pero por la escasa muestra utilizada (81 internos) no se podían asumir que los resultados sean confiables.

\section{Análisis factorial exploratorio de la prueba propiamente dicha (PPD)}

Se verificó la existencia, o no, de datos ausentes en la base de datos de los 23 ítems computables, posteriormente se identificó los datos atípicos y se corrigieron datos atípicos que fueron causa de un error de codificación. Se calcularon las puntuaciones típicas para las 23 variables; se evidenció que las variables 4, 6 y 10 tenían diversos casos de datos atípicos para puntuaciones estandarizadas. El método de box plots identificó datos atípicos, en las variables 4, 5, 6, 10, 11 y 12, pero se decidió retener los casos atípicos para experimentar y confirmar si se produce una afectación importante en la estructura factorial para la PPD. Se evaluó la distribución normal univariante a través de los estadísticos de asimetría y curtosis (Bandalos \& Finney, 2010; Gaskin, 2016; George \& Mallery, 2003; Lloret-Segura, Ferreres-Traver, Hernández-Baeza \& 
Tomás-Marco, 2014). Los ítems 4, 11 y 12 tenían índices de asimetría o curtosis más altos a 1.5, esto seguramente debido a los casos atípicos que se detectaron anteriormente en las mismas variables.

Para identificar la pertinencia de los datos de la muestra, se utilizó el Índice de medida de adecuación de la muestra KMO y el Test de esfericidad de Barlett (De la Fuente, 2011; Frías \& Pascual, 2012). Un índice de .846 para el coeficiente de KMO, el cual se considera bueno para realizar el AFE (Carmona, 2014; Káiser, 1974; Pett, Lackey \& Sullivan, 2003), y un Índice de Barlett, significativo, $p<0.01$ (Pett, Lackey $\&$ Sullivan, 2003; Mvududu \& Sink, 2014; Romero \& Ordoñez, 2015).

Teniendo como precedente la versión original de la escala y la opinión del autor original de la misma, se decidió realizar una serie de modificaciones en cuanto el uso de métodos estadísticos, es así que se forzó el modelo de la adaptación a distribuirse en tres factores específicos (violencia, psicopatía, reincidencia). Además, para identificar la matriz factorial de la PPD se incluyeron inicialmente los 17 items computables de la versión original $(5,7,11,13,14,15,16,17,18,19,20,21,22$, 23, 24, 25 y 26), más los items 4, 6,8,10,12 y 27; siendo el porcentaje de la varianza acumulada de 39.981 para los tres factores.

Esta se obtuvo a través del método de ejes principales, por cuanto este método disiminuye significativamente la varianza acumulada pero suele ser más adecuado y puro para el análisis en comparación de otros métodos. Se usó el método de ejes principales debido a la no normalidad de los datos de la muestra, y sobre todo porque este método analiza únicamente la varianza común, lo cual es considerado como principio fundamental para el AFC (Kline, 2011; Romero \& Ordoñez, 2015). La utilización del método de ejes principales en la adaptación intentó solucionar deficiencias procedimentales de la versión original en las que se utilizó inadecuadamente el método de reducción de variables denominado análisis de componentes principales, que está mal identificado como un método de análisis de factor común, ya que este no hace una diferenciación de la varianza única y la varianza común (Brown, 2015; Kline, 2011; Morales, 2013).

También se utilizó una rotación oblicua promax, al ser mejor que la rotación ortogonal usada en la versión original, denominada varimax 
que considera la independencia entre factores, lo cual es completamente incongruente en ciencias que investigan la conducta, sobre todo en psicología (Brown, 2015; Mulaik, 2009). Los ítems que formaron la estructura factorial de la adaptación se aprecian en la tabla 1; estos tienen saturaciones $>.30$ consideradas como significativas (Brown, 2015; Kline, 2011) y no tienen un peso similar o cercano en más de un solo factor, como se observa en la Tabla 1.

\section{Tabla 1}

Matriz de configuración factorial obtenida a través del método de ejes principales con rotación promax

\begin{tabular}{lccc}
\hline Variables & Vactor & \\
& Violencia & Reincidencia & Psicopatía \\
\hline Ítem 24 & .709 & & \\
Ítem 26 & .671 & & \\
Ítem 20 & .637 & & \\
Ítem 27 & .602 & & \\
Ítem 6 & .586 & & \\
Ítem 21 & .555 & & \\
Ítem 16 & .550 & & \\
Ítem 5 & .516 & & \\
Ítem 25 & .506 & & \\
Ítem 19 & .386 & & \\
Ítem 23 & .351 & .659 & .698 \\
Ítem 11 & & .695 & \\
Ítem 12 & & & \\
Ítem 13 & & & \\
Ítem 22 & & & \\
Ítem 17 & & & \\
Ítem 18 & & & \\
\hline
\end{tabular}

Nota: Se suprimieron los valores $\leq .3$ para una mejor apreciación del orden factorial. ${ }^{a}$ La rotación ha convergido en 5 iteraciones. 
La fiabilidad como consistencia interna del instrumento se evidenció a través del Alfa de Crombach; con un coeficiente de $\alpha=.837$, mostrando un buen índice de fiabilidad para la escala (Batista, Coenders \& Alonso, 2004; Romero \& Ordońez, 2015).

\section{Análisis factorial confirmatorio $(A F C)$ de la PPD}

Para el AFC se utilizó el software AMOS v.24, que trabaja con una interfaz gráfica creada en base a una hipótesis inicial del AFE y de la teoría que se maneja para la investigación. Se evidenció el indice de curtosis multivariante y su relación crítica (CR), que representa la estimación normalizada de Mardia (1985). El valor de la curtosis multivariante fue de .576 y su relación critica de .197 no proporcionó una curtosis significativa, lo que es indicativo de que los datos multivariantes se distribuyen de manera relativamente normal (Bentler, 2005).

Se especificaron tres variables factoriales latentes (violencia, reincidencia y psicopatía); 11 variables observables para el factor violencia, tres variables observables para el factor reincidencia y tres variables observables para el factor psicopatía. Los índices de ajuste fueron $\mathrm{CMIN} / \mathrm{df}=2.499, \mathrm{RMR}=.047, \mathrm{GFI}=.900, \mathrm{NFI}=.815, \mathrm{TLI}=.857$, $\mathrm{CFI}=.878$, RMSEA $=.070$; no siendo los más adecuados (Arbuckle, 2014; Bagozzi \& Yi, 2012; Brown, 2015; Chinda \& Mohamed, 2008; Doloi, Iyer \& Sawhney, 2010; Hu \& Bentler, 1998; Hair, Black, Babin \& Anderson, 2009; Mvududu \& Sink, 2014; Singh, 2009; Yu, 2002). Se re-especificó el modelo teniendo en cuenta los pesos regresión estandarizados, las correlaciones múltiples al cuadrado, los índices de modificación, la matriz de covarianzas residuales estandarizadas y los índices de ajuste del modelo.

Se re-especificaron las tres variables factoriales latentes; 9 variables observables para el factor violencia, tres variables observables para el factor reincidencia y tres variables observables para el factor psicopatía. Los índices de ajuste fueron; CMIN/df $=1.773, \mathrm{RMR}=.039$, $\mathrm{GFI}=.941, \mathrm{NFI}=.890, \mathrm{TLI}=.938, \mathrm{CFI}=.951, \mathrm{RMSEA}=.049$; siendo para el modelo re-especificado todos los índices de ajuste adecuados (Arbuckle, 2014; Bagozzi \& Yi, 2012; Brown, 2015; Chinda \& 
Mohamed, 2008; Doloi, Iyer \& Sawhney, 2010; Hu \& Bentler, 1998; Hair, Black, Babin \& Anderson, 2009; Mvududu \& Sink, 2014; Singh, 2009; Yu, 2002). La figura 1 muestra la re-especificación del modelo.

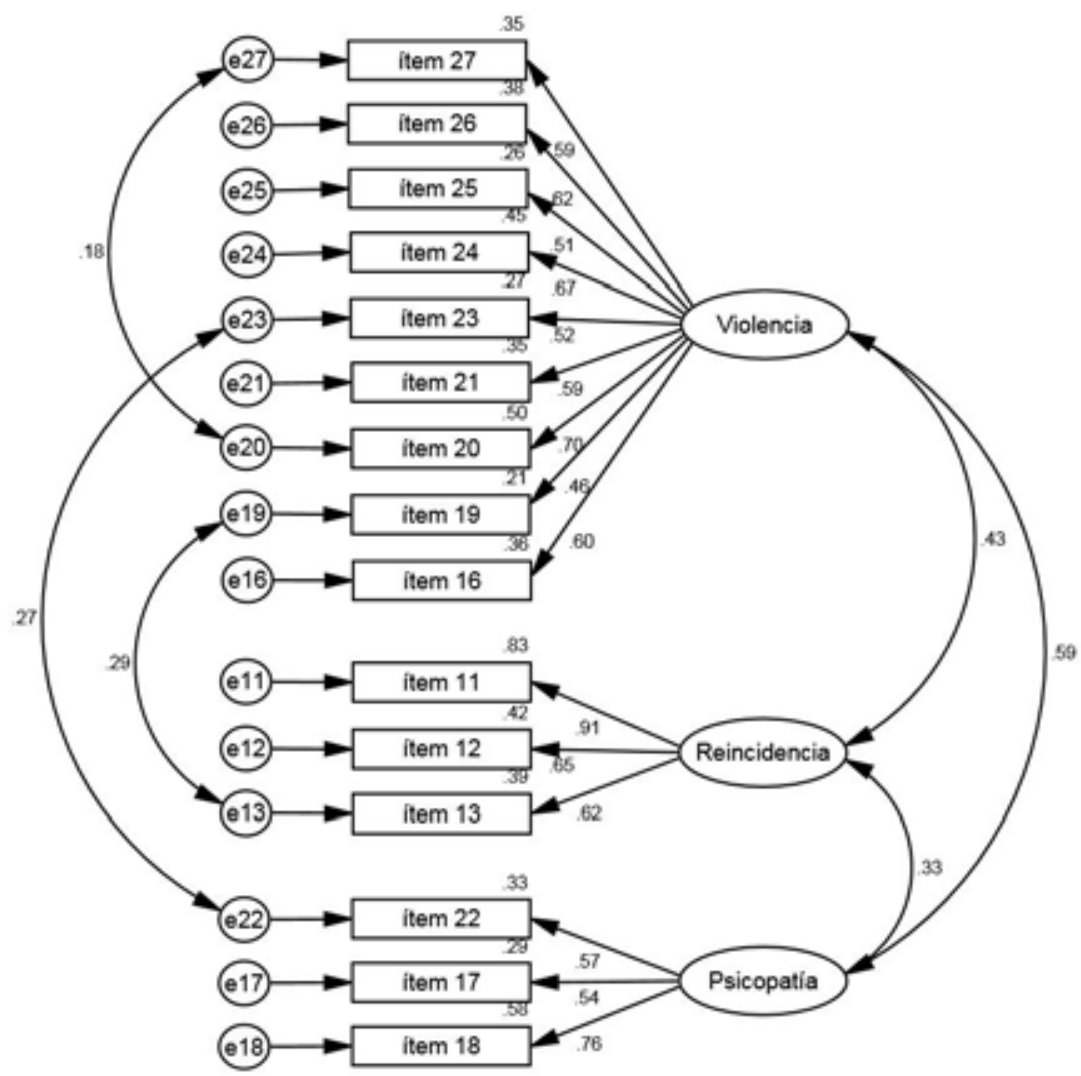

Figura 1. Diagrama de ruta y estimadores estandarizados para el modelo re-especificado 


\section{Precisión diagnóstica}

Se evaluó la precisión diagnostica de la adaptación de la EVCV-RR a través de la curva ROC (Receiver Operating Characteristic curve - ROC curve) con los participantes del presente estudio. Para esto, se utilizó el software MEDCALC v.17.2 (versión de prueba). Las puntuaciones para este procedimiento por un lado fueron la suma de los puntajes de los ítems de la escala y, por otro lado, el fundamento externo de identificación (violento/no-violento) para cada interno de la muestra, que fue concluido por la junta técnica de clasificación del establecimiento penitenciario del presente estudio, conformada por un psicólogo, un abogado y una trabajadora social.

La precisión diagnóstica, se midió a través de la curva ROC (Receiver Operating Characteristic curve - ROC curve) la cual representa tal vez el principal y preferido método de discriminación para precisión diagnóstica, (Hanson, 2009; Howard, 2017; Singh, Desmarais \& Van Dorn, 2013; Steyerberg et al., 2010). La figura 2 muestra las coordenadas del análisis ROC para la puntuación total de la escala.

El área bajo la curva de la escala total fue de AUC $=.910$, con un intervalo de confianza al $95 \%$, entre $.872-.940$ y estadísticamente significativa $p<0.0001$; el valor del AUC se interpreta como la probabilidad (91\%) de que un par de internos seleccionados aleatoriamente del grupo considerado como violento y del grupo considerado como no-violento sean clasificados adecuadamente tras aplicarles la escala y obtener un puntaje total. Además, este índice aporta a la fiabilidad de la escala, es decir, indica que el instrumento mide lo que pretende medir (Jiménez, F., comunicación personal, 28 de enero de 2017). Este análisis además muestra la sensibilidad, que refiere una probabilidad del $85.7 \%$ de clasificar correctamente a un sujeto como violento y también muestra la especifidad que se interpreta como la probabilidad de un $80.5 \%$ de clasificar correctamente a un sujeto no-violento. El punto de corte de este análisis, fue de $>20$. 


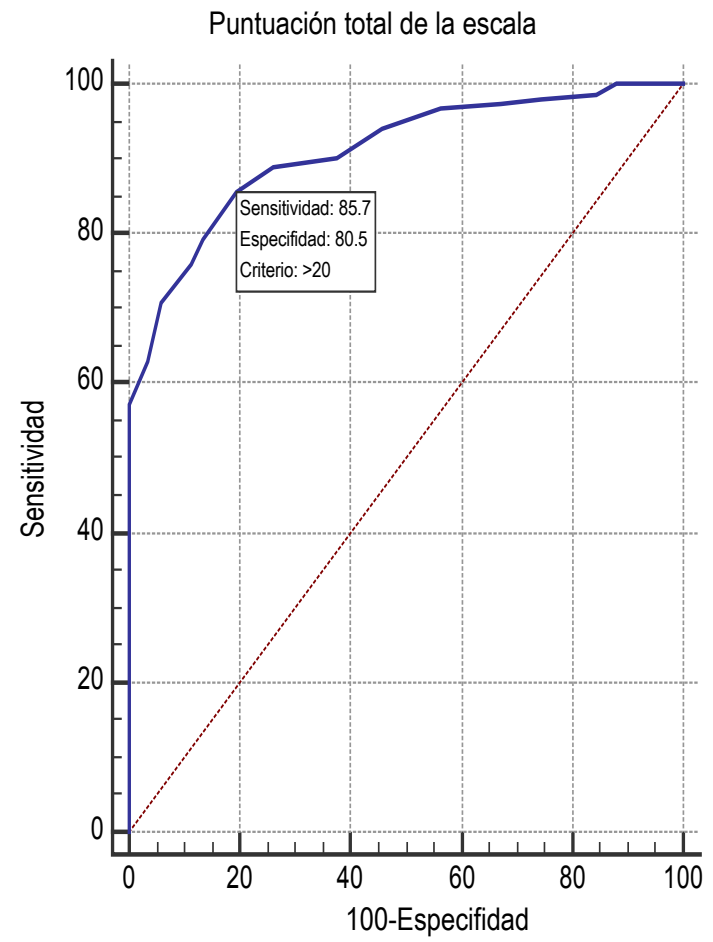

Figura 2. Coordenadas del análisis ROC para la escala total

La fiabilidad, como consistencia interna del instrumento, a través, del coeficiente Alfa de Cronbach para las 15 variables identificadas por el AFC fue de un $\alpha=.828$, lo cual indica una buena fiabilidad de la escala total.

Por último, se analizaron las diferencias entre las puntuaciones de las medias del grupo identificado como violento y no violento; $M=26.77 ; D E=6.206$, para el grupo considerado como violento y $M=17.19 ; D E=3.851$, para el grupo considerado como no-violento, obteniendo un tamańo del efecto para la muestra de 303 internos de $d=2.017$ considerándose un tamaño del efecto grande, según Cohen (1988). 


\section{Discusión}

Aunque en el mundo existen diversas metodologías de valoración del riesgo de conductas violentas en poblaciones penitenciarias, en el Perú no se utiliza a la actualidad ninguna de ellas; es por ello que la adaptación de la EVCV-RR resulta necesaria, por estar basada en las principales escalas, guías e instrumentos desarrollados en diferentes contextos que han ido fundamentando a través de los factores de riesgo estáticos y dinámicos, una base firme para poder crear instrumentos para valorar el riesgo de violencia (Conroy \& Murrie, 2007; Douglas \& Skeem, 2005; Douglas, Hart, Webster \& Belfrage, 2013; Hare, 2003; Hanson, 2004; Hanson \& Thornton, 2000; Jiménez et al., 2014b; Lewis, Olver \& Wong, 2016; Lucioni \& Pueyo, 2015; Monahan, et al., 2005, 2008; Quinsey, Harris, Rice \& Cormier, 1998, 2006; Schuringa, Heininga, Spreen \& Bogaerts, 2016; Webster, Douglas, Eaves \& Hart, 1997). La presente investigación está fundamentada en la metodología más actual que, a la luz de la literatura internacional, es la más adecuada para valorar una conducta criminal futura.

Aunque existe aún una pugna por demostrar la mayor eficacia entre instrumentos actuariales e instrumentos basados en el juicio clínico estructurado, esta investigación usa una combinación de métodos denominado Evaluación de riesgo estructurado (Monahan, 2008). Esta metodología parece ser aún más realista que otros métodos y además que múltiples autores como Esbec \& Fernández (2003); Heilbrun (2009); Jiménez et al. (2014b); Monahan (2008); Muñoz y López (2016); Pueyo y Arbach-Lucioni (2015); Quinsey et al. (2006) lo recomiendan.

Si bien es cierto que la presente investigación desarrolló la adaptación de la EVCV-RR para la realidad penitenciaria peruana y que, por ende, debería seguir la estructura lógica y metodológica con la cual se creó la escala original, se vio por conveniente junto al autor original de la escala modificar algunos procedimientos estadísticos a lo largo del desarrollo del instrumento con el fin de mejorar técnicamente la calidad del instrumento y su precisión. Existen diversas diferencias entre la versión original de la EVCV-RR y la adaptación peruana de la 
misma, de manera importante se decidieron mantener los tres factores de la escala original (violencia, psicopatía y reincidencia) y aunque estos no estuvieron compuestos por los mismo ítems en su totalidad, pero si por la mayoría, demostrando de manera real la solides de la conformación de los ítems para cada factor.

La adaptación de la EVCV-RR, a diferencia de la escala original, no usó el método de extracción de análisis de componente principales, aunque este método es preferido por los investigadores (Abad, Olea, Ponsoda \& García, 2011; Costello \& Osborne, 2005) suele no ser el más adecuado para muchas investigaciones de este tipo, ya que está mal identificado como un método de análisis de factor común (Brown, 2015), ya que sus métodos matemáticos son notablemente diferentes. En su lugar, esta investigación usó el método de ejes principales el cual es recomendable para datos de distribución no normal (como los que se obtuvieron en esta investigación), además de ser más conservadora ya que analiza únicamente la varianza común teniendo en cuenta la varianza de error de las variables (Kline, 2011).

Debido a la utilización del método de ejes principales se evidenció una disminución importante en el porcentaje acumulado de la varianza explicada por el modelo, pero esta a su vez resultó más pura, ya que mantuvo la lógica del modelo del factor común (Brown, 2015; Child, 2006; Kline, 2011; Morales, 2013). La rotación para el proceso de adaptación también fue diferente: en la versión original se utilizó un tipo de rotación ortogonal - varimax y en la adaptación peruana se utilizó un tipo de rotación oblicua - promax. Esta última rotación estimó que los factores estaban correlacionados en base al argumento de que en las investigaciones de ciencias sociales y conductuales siempre se evidenciará conceptualmente que los factores se correlacionan en alguna medida (Costello \& Osborne, 2005).

Además, se recomienda que cuando el AFE será un paso previo al AFC las rotaciones oblicuas son mejores a diferencia de las rotaciones ortogonales (Brown, 2015; Costello \& Osborne, 2005). Como acápite de lo anteriormente dicho, Byrne (2010) seńala que los índices no brindan necesariamente un indicador estricto de aceptación de un 
constructo, más bien que este tipo de discernimiento es responsabilidad del investigador, el cual debe basarse en un sustrato teórico sólido, la información estadística y sobre todo en la aplicabilidad del instrumento. Así un modelo puede ser útil en la práctica sin serlo estadísticamente (Arbuckle, 2014).

Por otra parte, algunos valores para las comunalidades de los ítems en las diferentes etapas de la adaptación peruana de la EVCV-RR fueron menores a $.30 \mathrm{y}$, aun así, fueron retenidos para insertarlos a los diversos procedimientos estadísticos. Esto debido a que lógicamente deberían formar parte de la escala, aunque estén aportando una baja varianza al factor común (Child, 2006; Gaskin, 2016). Estas comunalidades bajas mostraron, como consecuencia de su retención, algunas dificultades en el AFC de esta adaptación de la EVCV-RR y por consiguiente fueron eliminados con fundamento en la información brindada por los estadísticos índices de ajuste.

Los índices de ajuste para la adaptación peruana de la EVCV-RR, en su totalidad fueron considerados como adecuados a excepción del índice de ajuste chi-cuadrado, el cual no se consideró relevante, debido a que la muestra utilizada en esta adaptación es medianamente grande y esto afecta a la sobreestimación de este índice (Brown, 2015; Hu \& Bentler, 1998).

La versión original de la escala al igual que la adaptación están conformada por 27 ítems, de los cuales 17 ítems han sido definidos como computables en la versión original y 15 han sido definidos como computables para la adaptación peruana. Los demás items para ambas versiones son de información complementaria importante para el evaluador. Todos estos ítems tienen un adecuado respaldo de la teoría e investigaciones en el desarrollo de diversos instrumentos basados en el método clínico estructurado tal como el HCR-20 v.3, de Douglas, Hart, Webster \& Belfrage, (2013) y actuarial como es el VRAG, de Quinsey et al. (1998, 2006), VRAG-R de Rice, Harris \& Lang (2013) y la PCL-R, 2da. Ed. de Hare (2003). La adaptación de la EVCV$\mathrm{RR}$ además muestra diferencias con otros instrumentos de amplio uso como son el HCR-20, VRAG, VRAG-R y la PCL-R. 
La primera diferencia de la adaptación peruana es que es de uso exclusivo para población penitenciaria. La segunda diferencia es que este instrumento usa metodología clínica estructurada (Monahan, 2008), al basarse en una guía de variables que son valoradas por el entrevistador y una metodología actuarial al usar una valoración a través de puntuaciones para cada ítem, para cada sub-escalas y la escala total. La tercera diferencia es que esta escala es de uso general penitenciario, no es específica para un tipo especial de violencia como por ejemplo el SVR-20 lo es para la violencia sexual (Boer, Hart, Kropp \& Webster, 1997; Pueyo \& Hilterman, 2005).

Los 15 ítems computables de la adaptación peruana de la EVCV-RR obtuvieron una consistencia interna de $\alpha=.828$ y una precisión diagnostica de AUC = .910. En comparación, la escala original (primeros resultados) estuvo conformada por una muestra de 49 internos en un centro penitenciario de Espańa; la consistencia interna fue de $(\alpha=.835)$ y la precisión diagnóstica (AUC = .904) (Jiménez et al., 2014). La versión original, como la adaptación en una muestra penitenciaria peruana, obtuvieron buenos índices de confiabilidad y del AUC, aunque la diferencia de tamaños de muestra es muy marcada y la versión original no se acercó a una muestra mínima para un AF; esto hace suponer que los ítems dieron solidez a las diferentes versiones; identificando para ambas versiones las mismas sub-escalas (violencia, psicopatía y reincidencia), aunque tuvieron variaciones en la distribución de ítems, pero permaneció la solidez lógica y teórica en la adaptación con una muestra peruana.

Los puntos de corte para ambas adaptaciones, para cada sub-escala y para el puntaje total, se dividió en cinco niveles (muy bajo, bajo, moderado, alto y muy alto) para una mejor comprensión y diferenciación de la valoración. Además, se nota una diferencia entre baremos, obteniendo los baremos peruanos puntos de corte con puntuaciones más altas en comparación con los puntos de cortes para las puntuaciones espańolas; aparentemente debido a que la muestra peruana parece ser más violenta que la muestra española.

Por otra parte, cabe mencionar limitaciones en esta investigación, tales como el hecho de que no se haya tenido una muestra nacional ya 
que es difícil obtener información de los 63 penales peruanos (regímenes ordinarios y especiales) para obtener mayor variabilidad de datos que aporten a establecer estructuras factoriales mejor definidas, además de poder desarrollar una versión aplicable a internas mujeres. Otro problema importante fue que en nuestro país no existían investigaciones sobre adaptaciones o de desarrollo de instrumentos en varones dentro del ámbito penitenciario, lo cual complicó de manera importante el inicio de esta investigación. Todas las limitaciones antes mencionadas pueden resolverse sobre todo con apoyo integro de instituciones como el INPE, Ministerio de Justicia, universidades e institutos de investigación que enfoquen sus intereses y de forma clara su presupuesto en el desarrollo de investigaciones que brinden soluciones a problemas reales como son las conductas delictivas en nuestro país.

Finalmente, la contribución de la adaptación peruana de la EVCV$\mathrm{RR}$ es brindar el primer instrumento estructurado específico para valorar el riesgo criminógeno en la población penitenciaria peruana, la misma que en la práctica dota actualmente de eficacia a los profesionales penitenciarios al momento de respaldar sus decisiones cuando se trata de redactar algún informe y ratificarlo ante instancia judicial o penitenciaria sobre la valoración del riesgo criminógeno de algún interno, clasificarlo y trasladarlo a otro penal (es de importancia mencionar que el instrumento viene siendo aplicado en los penales de Socabaya Arequipa, Pocollay - Tacna, Castro Castro - Lima y Cachiche - Ica, por psicólogos de amplia experiencia en penales, los cuales muestran gran aceptación y recomendación de la adaptación de la EVCV-RR al ámbito penitenciario peruano). Esta herramienta, específicamente ayuda en la valoración de otorgamiento o negación de los beneficios penitenciarios de semi-libertad y liberación condicional, además de ser funcional en diversas circunstancias relacionadas a la evaluación del comportamiento delictual entre ellas interceder con la problemática del hacinamiento en los establecimientos penitenciarios. Asimismo, el desarrollo de esta investigación también aportará como medida de evaluación para distintas investigaciones en conducta criminal en el área de la psicología, el derecho, la sociología y otras especialidades, 
siendo uno de los puntos principales el comenzar una tendencia para el desarrollo de instrumentos y técnicas bajo estricta metodología, procedimientos adecuados y válidos para el campo de la psicológica forense, penitenciaria y criminal.

\section{Referencias}

Abad, F., Olea, J., Ponsoda, V. \& García, C. (2011). Medición en ciencias sociales y de la salud. Madrid, España: Síntesis.

Aiken, L. R. (2003). Test psicológicos y evaluación. México: Pearson Education.

Andrade, J. T., O'neill, K. \& Diener R.B. (2009). Violence risk assessment and risk management: a historical overview and clinical application. En J. T. Andrade (Ed.), Handbook of Violence Risk Assessment and Treatment (pp. 3-39). Nueva York: Springer Publishing Company.

Andrews, D. A., Bonta, J. \& Wormith, S. J. (2006). The recent past and near future of risk and /or need assessment. Crime and Delinquency, 52, 7-27. https://doi.org/10.1177/0011128705281756

Arbach-Lucioni, K., Desmarais, S. L., Hurducas, C., Condemarin, C., Dean, K., Doyle,... Singh, J. (2015). La práctica de la evaluación del riesgo de violencia en España. Revista de la Facultad de Medicina, 63(3), 357-366. https://doi.org/10.15446/revfacmed. v63n3.48225

Arbuckle, J. L. (2014). Amos v.23 user's guide. Chicago: IBM SPSS

Bagozzi, R. \& Yi, Y. (2012). Specification, evaluation, and interpretation of structural equation models. Journal of the Academy of Marketing Science, 40, 8-34.

Bandalos, D. L. \& Finney, S. J. (2010). Factor analysis: Exploratory and confirmatory. En G. R. Hancock y R. O. Mueller (Eds.), Reviewer's guide to quantitative methods (pp. 93-114). Nueva York: Routledge. 
Batista, J. M., Coenders, G. \& Alonso, J. (2004). Análisis factorial confirmatorio. Su utilidad en la validación de cuestionarios relacionados con la salud. Medicina Clínica, 122(1), 21-27.

Beavers, A., Lounsbury, J., Richards, J., Huck, Sh., Skolits, G. \& Esquivel, S. (2013). Practical considerations for using exploratory factor analysis in educational research. Practical Assessment, Research \& Evaluation, 18(6). Recuperado de: http://www.pareonline.net/getvn.asp?v=18\&n=6

Bentler, P. M. (2005). EQS VI Structural equations program manual. Encino, CA: Multivariate Software.

Boer, D., Hart, S., Kropp, R. \& Webster, C. (1997). Manual for the Sexual Violence Risk-20. Burnaby, British Columbia, Canada: Simon Fraser University, Mental Health, Law, and Policy Institute.

Bonta, J., Law, M. \& Hanson, K. (1998). The prediction of criminal and violent recidivism among mentally disordered offenders: A meta-analysis. Psychological Bulletin, 123, 123-142.

Brown, J. \& Singh, J. P. (2014). Forensic risk assessment: A beginner's guide. Archives of Forensic Psychology, 1(1), 49-59.

Brown, T. (2015). Confirmatory factor analysis for applied research (2nd Ed.). Nueva York: The Guilford Press.

Byrne, B. (2010). Structural equation modeling with AMOS. Basic concepts, applications, and programming (2nd. Ed.). Nueva York: Routledge, Taylor \& Francis Group.

Carmona, F. (2014). Un ejemplo de ACP paso a paso. Departament d'Estadistica. Universitat de Barcelona. Recuperado de: http:// www.ub.edu/stat/docencia/Mates/ejemploACP.PDF

Child, D. (2006). The essentials of factor analysis. (3rd Ed.). Nueva York: Continuum International Publishing Group.

Chinda, T. \& Mohamed, S. (2008). Structural equation model of construction safety culture. Engineering, Construction and Architectural Management, 15(2), 114-131.

Cohen, J. (1988). Statistical power analysis for the behavioral sciences (2nd Ed.). New Jersey: LEA 
Conroy, M. A. \& Murrie, D. C. (2007). Forensic assessment of violence risk: A guide for risk assessment and risk management. Hoboken, NJ: Wiley. https://doi.org/10.1002/9781118269671

Costello, A. \& Osborne, J. (2005). Best practices in exploratory factor analysis: Four recommendations for getting the most from your analysis. Practical Assessment Research \& Evaluation, 10(7), 1-9. Recuperado de: http://pareonline.net/getvn.asp?v=10\&n=7

De Borba, L. E., Folino, J. O. \& Taborda, J. G. (2009). Incidência de conduta violenta e antissocial em população psiquiátrica forense. Revista de Psiquiatría de Rio Grande do Sul, 33(1), 3-7.

De la Fuente, S. (2011). Análisis factorial. Madrid, España: Universidad Autónoma de Madrid.

Decreto legislativo $\mathrm{n}^{\circ} 1328$, art. 30. Decreto legislativo que fortalece el sistema penitenciario nacional y el Instituto Nacional Penitenciario. Lima, Perú. (6 de enero del 2017).

Department of Family and Community Services. (2015). Violence risk assessment practice guide. Practice guide for practitioners who support people with disability (v 1.0). New South Wales, Australia.

Doloi, H., Iyer, K. \& Sawhney, A. (2010). Structural equation model for assessing impacts of contractor's performance on project success. International Journal of Project Management, 29, 687-695.

Douglas, K. S. \& Skeem, J. L. (2005). Violence risk assessment: Getting specific about being dynamic. Psychology, Public Policy, and Law, 11(3), 347-383. https://doi.org/10.1037/1076-8971.11.3.347

Douglas, K. S., Hart, S. D., Webster, C. D. \& Belfrage, H. (2013). HCR-20 v.3. Assessing risk for violence. Burnaby, Canada: Mental Health, Law, and Policy Institute, Simon Fraser University.

Esbec, E. \& Fernández O. (2003). Valoración de la peligrosidad criminal (riesgo-violencia) en psicología forense. Instrumentos de evaluación y perspectivas. Psicopatología Clínica, Legal y Forense, 3(2), 65-90.

Fazel, S., Singh, J. P., Doll, H. \& Grann, M. (2012). Use of risk assessment instruments to predict violence and antisocial behaviour in 73 samples involving 24827 people: Systematic Review and 
Meta-Analysis. BMJ, 345, e4692. https://doi.org/10.1136/bmj. e4692

Fernández-Ballesteros, R., Oliva, M., Vizcarro, C. \& Zamarrón, M. D. (2011). Buenas prácticas y competencias en evaluación psicológica. Madrid: Pirámide.

Folino, J. O. \& Hare, R. D. (2005). Listado revisado para verificación de la psicopatía: su estandarización y validación en la Argentina. Acta Psiquiátrica y Psicológica de América, 51(2), 94-104.

Folino, J. O. (2000). Una subespecialización psiquiátrica: La psiquiatría forense. En M. Suárez (Ed.). Introducción a la Psiquiatría (2, pp. 441-449). Buenos Aires: Salerno.

Folino, J. O. (2015). Predictive efficacy of violence risk assessment instruments in Latin-America. The European Journal of Psychology Applied to Legal Context, 7, 51-58. https://doi.org/10.1016/j. ejpal.2014.11.006

Folino, J. O., Sarmiento, D. R. \& Montero, V. J. (2000). Forensic system in the province of Buenos Aires, Argentina. International Journal of Law and Psychiatry, 23, 567-578.

Frías, D. \& Pascual, M. (2012). Practicas del análisis factorial exploratorio (AFE) en la investigación sobre conducta del consumidor y marketing. Suma Psicológica, 19(1), 47-58.

Fuster, J. M. (2014). Cerebro y libertad. Barcelona: Ariel. https://doi. org/10.1016/S0160-2527(00)00060-1

Gaskin, J. (2016). Exploratory factor analysis. Recuperado de: http://statwiki.kolobkreations.com/index. php?title=Exploratory_Factor_Analysis

George, D. \& Mallery, M. (2003). Using SPSS for Windows step by step: A simple guide and reference. Boston, MA: Allyn \& Bacon.

Grann, M., Belfrage, H. \& Tengström, A. (2000). Actuarial assessment of risk for violence: Predictive validity of the VRAG and the historical part of the HCR-20. Criminal Justice and Behavior, 27, 97-114. https://doi.org/10.1177/0093854800027001006

Hair, J. F., Black, W. C., Babin, B. J. \& Anderson, R. E. (2009). Multivariate data analysis (7th Ed.). Nueva York, EE.UU: Pearson. 
Hanson, R. \& Thornton, D. (2000). Improving risk assessments for sex offenders: A comparison of three actuarial scales. Law Hum Behav, 24, 119-136. https://doi.org/10.1023/A:1005482921333

Hanson, R. (2004). The development of a brief actuarial scale for sexual offense recidivism. Ottawa, Canada: Department of the Solicitor General of Canada.

Hanson, R. (2009). The psychological assessment of risk for crime and violence. Canadian Psychology, 50(3), 172-182. https://doi. org/10.1037/a0015726

Hare, R. D. (2003). The Hare Psychopathy Checklist-Revised (2nd Ed.). Toronto, Canada: Multi-Health Systems.

Harris, G. T. \& Rice, M. E. (2007). Adjusting actuarial violence risk assessments based on aging or the passage of time. Criminal Justice and Behavior, 34(3), 297-313. https://doi. org/10.1177/0093854806293486

Hart, C., Michie, S. \& Cooke, D. (2007). Precision of actuarial risk assessment instruments: evaluating the 'Margins of Error' of group V. Individual predictions of violence. British Journal of Psychiatry, 190(49), 60-65. https://doi.org/10.1192/bjp.190.5.s60

Heilbrun, K. (2009). Evaluation for risk of violence in adults. Nueva York: Oxford University Press, Inc. https://doi.org/10.1093/ med:psych/9780195369816.001.0001

Helmus, L. \& Babchishin, K. (2017). Primer on risk assessment and the statistics used to evaluate its accuracy. Criminal Justice and Behavior, 44(1), 8-25. https://doi.org/10.1177/0093854816678898

Hernández, R., Fernández, C. \& Baptista, P. (2014). Metodología de la investigación. Gta. Ed. México D.F., México: McGraw-Hill.

Howard, P. D. (2017). The effect of sample heterogeneity and risk categorization on area under the curve predictive validity metrics. Criminal Justice and Behavior, 44(1), 103-120. https:// doi.org/10.1177/0093854816678899

Hu, L. \& Bentler, P. M. (1998). Fit indices in covariance structure modeling: sensitivity to underparameterized model 
misspecification. Psychological Methods, 3, 424-453. https://doi. org/10.1037/1082-989X.3.4.424

Instituto Nacional Penitenciario (2016). Informe estadístico penitenciario (julio-2016). Recuperado de: http://www.inpe.gob.pe/ pdf/julio_2016.pdf

Jiménez, F., Sánchez, G., Merino, V. \& Ampudia, A. (2014a). Escala de valoración de la conducta violenta y riesgo de reincidencia (EVCV-RR): Primeros resultados. Revista Iberoamericana de Diagnóstico y Evaluación - e Avaliação Psicológica, 2(30), 87-104. Jiménez, F., Sánchez, G., Merino, V. \& Ampudia, A. (2014b). Evaluación de la peligrosidad en la población penitenciaria. Valoración del riesgo de violencia y su reincidencia. Salamanca: Ratio Legis.

Káiser, F. H. (1974). An index of factorial simplicity. Psychometrika, 39(1), 31-36. Recuperado de: http://jaltcue.org/files/articles/ Káiser1974_an_index_of_factorial_simplicity.pdf

Kline, R. B. (2011). Principles and practice of structural equation modeling (3ra Ed.). Nueva York: Guilford.

Kroner, D. G. (2005). Issues in violent risk assessment. Lessons learned and future directions. Journal of Interpersonal Violence, 20(2), 231-235. https://doi.org/10.1177/0886260504267743

León, E., Asún, D., Folino, J. (2010). Confiabilidad y validez de la versión chilena de la Hare PCL-R. Revista de la Facultad de Medicina, 58(2), 103-114.

Lewis, K., Olver, M. E. \& Wong, S. C. P. (2016). The violence risk scale: Predictive validity and linking changes in risk with violent recidivism in a sample of high-risk offenders with psychopathic traits. Assessment, 20(2), 150-164. https://doi. org/10.1177/1073191112441242

Lloret-Segura, S., Ferreres-Traver, A., Hernández-Baeza, A. \& TomásMarco, I. (2014). El análisis factorial exploratorio de los ítems: una guía práctica, revisada y actualizada. Anales de Psicología, 30(3), 1151-1169. https://doi.org/10.6018/analesps.30.3.199361 
Louw, D. A., Strydom, C. C. \& Esterhuyse, K. G. F. (2005). Prediction of violent behaviour: Professionals' appraisal. Criminal justice, 5(4), 379-406. https://doi.org/10.1177/1466802505057717

Loyola, N. (2011). Validez y confiabilidad de la Escala de Impulsividad de Barratt Versión 11 (BIS-11) en mujeres encarceladas (Tesis de pregrado) Pontificia Universidad Católica del Perú. Lima, Perú.

Lucioni, K. A \& Pueyo, A. A. (2015). HCR-20 v.3 Valoración del riesgo de violencia. Barcelona: Edicions de la Universitat de Barcelona.

Mardia, K. V. (1985). Mardia's test of multinormality. En S. Kotz y N. L. Johnson (Eds.), Encyclopedia of statistical sciences (5, pp. 217-221). Nueva York: Wiley.

Martínez, L. (2014). La incertidumbre de los pronósticos de peligrosidad: Consecuencias para la dogmática de las medidas de seguridad. Revista para el Análisis del Derecho, 2(14), 1-77.

Medina, P., Valdés, M., Galán, L., Vergara S. \& Couso, O. (2013). Confiabilidad y validez de la Escala de Psicopatía Revisada de Hare (PCL-R) en la población carcelaria cubana. Salud Mental, 36(6), 481-486. https://doi.org/10.17711/SM.0185-3325.2013.058

Merino, C. \& Livia, J. (2009). Intervalos de confianza asimétricos para el índice de validez de contenido: Un programa Visual Basic para la V de Aiken. Anales de Psicología, 25(1), 169-171. Recuperado de http://revistas.um.es/analesps/article/view/71631/69111

Moltó, J., Poy, R. \& Torrubia, R. (2000). Standardization of the Hare Psychopathy Checklist. Revised in a spanish prison simple. Journal of Personality Disordes, 14, 84-96. https://doi. org/10.1521/pedi.2000.14.1.84

Monahan, J. (2003). Violence risk assessment. En A. M. Goldstein (Ed.), Handbook of Psychology (11, pp. 527-540). Hoboken, New Jersey: Wiley. https://doi.org/10.1002/0471264385.wei1126

Monahan, J. (2008). Structured risk assessment of violence. En R. Simon \& K. Tardiff (Eds.), Textbook of Violence Assessment and Management (pp. 17-33). Arlington, VA: American Psychiatric Publishing, Inc. 
Monahan, J., Steadman, H. J., Silver, E., Appelbaum, P. S., Clark Robbins, P., Mulvey, E. P.,... Banks, S. M. (2001). Rethinking Risk Assessment: The MacArthur study of mental disorder and violence. Nueva York: Oxford University Press.

Monahan, J., Steadman, H., Robbins, P. C., Appelbaum, P., Banks, S., Grisso, T.,... Silver, E. (2005). Prospective validation of the multiple iterative classification tree model of violence risk assessment. Psychiatric Services, 56, 810-815. https://doi. org/10.1176/appi.ps.56.7.810

Morales P. (2013) El Análisis Factorial en la construcción e interpretación de tests, escalas y cuestionarios. Madrid: Universidad Pontificia Comillas.

Mulaik, S. A. (2009). Foundations of factor analysis (2nd Ed.). Boca Raton, FL: Chapman \& Hall/ CRC.

Muñoz, J. M. \& López, J. J. (2016). Valoración psicológica del riesgo de violencia: Alcance y limitaciones para su uso en el contexto forense. Anuario de Psicologia Jurídica, 26(1), 130-140. https:// doi.org/10.1016/j.apj.2016.04.005

Mvududu, N. H. \& Sink, C. A. (2014). Factor analysis in counseling research and practice. Counseling Outcome Research and Evaluation, 4(2), 75-98.

Neal, T. M. S. \& Grisso, T. (2014). Assessment practices and expert judgment methods in forensic psychology and psychiatry: An international snapshot. Criminal Justice and Behavior, 41(12), 1406-1421. https://doi.org/10.1177/0093854814548449

Ostrosky, F., Ruiz, A., Arias, N. \& Vásquez, V. (2008). Estandarización de la PCL-R en población penitenciaria mexicana. Revista de Neuropsicología, Neuropsiquiatría y Neurociencias, 8(2), 49-58.

Otto, R. K. (2000). Assessing and managing violence risk in outpatient settings. JournalofClinical Psychology, 56(10), 1239-1262. https:// doi.org/10.1002/1097-4679(200010)56:10<1239::AIDJCLP2>3.0.CO;2-J 
Petrosino, A., Derzon, J. \& Lavenberg, J. (2009). The role of the family in crime and delinquency: Evidence from prior quantitative reviews. Southwest Journal of Criminal Justice, 6(2), 108-132.

Pett, M., Lackey, N. \& Sullivan, J. (2003). Making sense of factor analysis: The use of factor analysis for instrument development in health care research. Thousand Oaks, CA: Sage.

Pueyo, A. \& Arbach-Lucioni, K. (2015). Peligrosidad y valoración del riesgo de violencia en contextos forenses. En E. GarcíaLópez (Ed.), Manual de Psicopatología Forense: Comportamiento Humano y Tribunales de Justicia, (pp. 505-525). México DF: Manual Moderno.

Pueyo, A. \& Hilterman, E. (2005). SVR-20, Manual de valoración de riesgo de violencia sexual. Barcelona: Publicacions i Edicions.

Pueyo, A. \& Redondo, S. (2004). Aportaciones psicológicas a la predicción de la conducta violenta, reflexiones y estado de la cuestión. II Congreso Virtual de Psicología Jurídica y Forense. Madrid.

Pueyo, A. \& Redondo, S. (2007). Predicción de la violencia: Entre la peligrosidad y la valoración del riesgo de violencia. Papeles del Psicólogo, 28(3), 157-173.

Quinsey, V. L., Harris, G. T., Rice, M. E. \& Cormier, C. A. (1998). Violent offenders: Appraising and managing risk. The law and public policy: Psychology and the Social Sciences series. Washington, DC: American Psychological Association.

Quinsey, V. L., Harris, G. T., Rice, M. E. \& Cormier, C. A. (2006). Violent Offenders: Appraising and Managing Risk (2nd Ed.). Washington, DC: American Psychological Association. https:// doi.org/10.1037/11367-000

Rice, M. E., Harris, G. T. \& Lang, C. (2013). Validation of and revision to the VRAG and SORAG: The Violence Risk Appraisal Guide-Revised (VRAG-R). Psychological Assessment, 25(3), 951965. https://doi.org/10.1037/a0032878

Rodríguez, J. (2014). Analizando la V de Aiken usando el método score con hojas de cálculo [Hoja de cálculo]. Universidad de Cádiz, España. 
Romero, S. J. \& Ordoñez, X. G. (2015). Psicometría. Madrid, España: COYVE S.A.

Ruiz, J. (2006). Adaptación de la escala PCL-R 20 para evaluación de rasgos psicopáticos en una muestra carcelaria. Avances en Medición, 4, 61-72.

Schick, A. \& Cierpka, M. (2016) Risk factors and prevention of aggressive behavior in children and adolescents. Journal for Educational Research Online 8(1), 90-109. Recuperado de: http:// www.j-e-r-o.com/index.php/jero/article/view/623/260

Schuringa, E., Heininga, V. E., Spreen, M. \& Bogaerts, S. (2016). Concurrent and predictive validity of the instrument for forensic treatment evaluation: From risk assessment to routine, multidisciplinary treatment evaluation. International Journal of Offender Therapy and Comparative Criminology, 1-19.

Singh, J. P. (2009). Does my structural model represent the real phenomenon? A review of the appropriate use of structural equation modelling (SEM) Model Fit Indices. The Marketing Review, 9(3), 199-212.

Singh, J. P. (2012). The History, Development, and Testing of Forensic Risk Assessment Tools. En E. Grigorenko (Ed.), Handbook of Juvenile Forensic Psychology and Psychiatry (pp. 215-225). Nueva York: Springer. https://doi.org/10.1007/978-1-4614-0905-2_14 Singh, J. P., Condemarin, C., Folino, J. O. \& the IRiS Group (2013). El uso de instrumentos de evaluación de riesgo de violencia en Argentina y Chile. Revista Criminalidad, 55, 279-290.

Singh, J. P., Desmarais, S. D. \& Van Dorn, R. A. (2013). Measurement of predictive validity in violence risk assessment studies: A second-order systematic review. Behavioral Sciences and the Law, 31, 55-73. https://doi.org/10.1002/bsl.2053

Singh, J., Fazel, S., Gueorguieva, R. \& Buchaman, A. (2014). Rates of violence in patients classified as high risk by structured risk assessment instruments. The British Journal of Psychiatry, 204, 180-187. https://doi.org/10.1192/bjp.bp.113.131938 
Skeem, J. L. \& Monahan, J. (2011). Current directions in violence risk assessment. Current Directions in Psychological Science, 20(1), 38-42. https://doi.org/10.1177/0963721410397271

Steyerberg, E. W., Vickers, A. J., Cook, N. R., Gerds, T., Gonen, M., Obuchowski, N., Pencina, M. J. \& Kattan, M. W. (2010). Assessing the performance of prediction models: A framework for traditional and novel measures. Epidemiology, 21, 128-138. https://doi.org/10.1097/EDE.0b013e3181c30fb2

Tabachnick, B. \& Fidell, L. (2001). Using multivariate statistics. Needham Heights: Allyn \& Bacon.

Tapias, A. (2011). Aplicación de los instrumentos de reincidencia en violencia HCR-20 y SVR-20 en dos grupos de delincuentes colombianos. Revista Criminalidad, 53(1), 307-327. Recuperado de: http://www.scielo.org.co/pdf/crim/v53n1/v53n1a08.pdf

Tardiff, K. (2008). Clinical risk assessment of violence. En R. Simon \& K. Tardiff(Eds.), Textbook of Violence Assessment and Management (pp. 3-16). Arlington VA: American Psychiatric Publishing, Inc. Telles, L. E. de B., Day, V. P., Folino, J. O. \& Taborda, J. G. (2009). Reliability of the brazilian version of HCR-20, assessing risk for violence. Revista Brasileira de Psiquiatria, 31, 253-256. https:// doi.org/10.1590/S1516-44462009005000001

Tengströn, A., Hodings, S., Grann, M., Lángström, N. \& Kullgren, G. (2004). Schizophrenia and criminal offending: The role of psychopathy and substance use disorders. Criminal Justice and Behavior, 31, 367-391. https://doi. org/10.1177/0093854804265173

Webster, C. D., Douglas, K. S., Eaves, D. \& Hart, S. D. (1997). HCR-20 Assessing Risk for Violence (Version 2). Burnaby, British Columbia, Canada: Simon Fraser University, Mental Health, Law and Policy Institute.

Willmot, P. (2004). Applying the research on reducing recidivism to prison regimes. En G. Towl (Ed.), Psychology in prisons (pp. 35-51). Oxford: Blackwell Publishing Ltd. 
Wong, S., Olver, M. \& Stockdale, K. (2009). The utility of dynamic and static factors in risk assessment, prediction, and treatment. En J. T. Andrade (Ed.), Handbook of Violence Risk Assessment and Treatment (pp. 3-39). Nueva York: Springer Publishing Company.

Yu, C. Y. (2002). Evaluating cutoff criteria of model fit indices for latent variable models with binary and continuous outcomes (Tesis de Doctorado). University of California, LA.

Recibido: 5 de junio, 2017 Revisado: 6 de noviembre, 2017 Aceptado: 31 de enero, 2018 